Algebraic 8 Geometric $\mathcal{T}$ opology

Volume 5 (2005) 1173-1195

Published: 18 September 2005

ATG

\title{
Overtwisted open books from sobering arcs
}

\author{
NOAH GOODMan
}

\begin{abstract}
We study open books on three manifolds which are compatible with an overtwisted contact structure. We show that the existence of certain arcs, called sobering arcs, is a sufficient condition for an open book to be overtwisted, and is necessary up to stabilization by positive Hopf-bands. Using these techniques we prove that some open books arising as the boundary of symplectic configurations are overtwisted, answering a question of Gay in [9].
\end{abstract}

AMS Classification 57R17; 57M99

Keywords Open book, contact structure, overtwisted, sobering arc, symplectic configuration graph

\section{Introduction}

A contact structure on a three-manifold is a nowhere integrable two-plane field (we consider only oriented, co-orientable, positive contact structures). A fundamental theorem of Eliashberg [3] shows that contact structures fall into two classes: overtwisted, and tight. The overtwisted contact structures are determined by their homotopy type as plane fields, while the tight ones are more subtle.

An open book is a pair $(\Sigma, \phi)$, where $\Sigma$ is a surface with boundary, and $\phi$, the monodromy, is an automorphism of $\Sigma$. The mapping torus $\Sigma \times_{\phi} S^{1}$ has torus boundary with preferred meridian and longitude, we can thus form a three manifold $M_{(\Sigma, \phi)}$ by gluing solid tori to the boundary. We say that $(\Sigma, \phi)$ is an open book on $M_{(\Sigma, \phi)}$, each $\Sigma \subset M_{(\Sigma, \phi)}$ is a page, while the core of the solid tori are the binding.

A contact structure and an open book on the same manifold are called compatible if the contact planes can be isotoped arbitrarily close to the tangent planes of the pages, while the binding remains transverse. For example the standard tight contact structure on $S^{3}$, written $\xi_{0}$, is compatible with the positive Hopfband, $H^{+}$, whose binding is two fibers of the Hopf fibration. 
If we have two contact structures compatible with the same open book it is not hard to show (by first pushing the contact planes close to the pages, then using a straight-line homotopy) that they must be isotopic. (We will write $\xi_{B}$ for a contact structure compatible with an open book $B$, unique up to isotopy.) Because of this uniqueness the contact structures compatible with a given open book are either all tight or all overtwisted.

Definition 1.1 An open book $(\Sigma, \phi)$ is called overtwisted if there is an overtwisted contact structure $\xi_{(\Sigma, \phi)}$ compatible with it.

Giroux has shown [1] that there is an open book compatible with any contact structure, so it is natural to study contact structures by studying open books. The first question one would like to answer is: when is an open book overtwisted?

A few open books can be shown to be overtwisted by general considerations or special techniques. For example, every contact structure on the Poincare homology-sphere with reversed orientation is overtwisted [6], so any compatible open book must also be overtwisted. This manifold arises as +1 -surgery on the right-handed trefoil $T^{+}$, so it has an open book which comes from the natural open book structure of $T^{+}$by adding a boundary parallel negative Dehn twist. In section 3 we'll show more directly that this open book is overtwisted.

In this paper we study arcs (which we call sobering arcs) which intersect their monodromy images in specific ways. We show that the existence of a sobering arc is a sufficient condition for an open book to be overtwisted. This is not a necessary condition (there are overtwisted open books with no sobering arc), but it is necessary up to stabilization (by Murasugi sum with positive Hopfbands). The most satisfying statement of our result in this paper is:

Theorem 1.2 An open book is overtwisted if and only if it is stably equivalent to an open book with a sobering arc.

This technique gives some interesting examples: we look at the open books that arise from positive surface configurations in symplectic manifolds, studied by Gay in [9], and show that some of these open books are overtwisted, and hence the corresponding symplectic configuration cannot embed in a closed symplectic 4-manifold (answering a question of [9]).

In section 2 we review some necessary background material on contact structures and open books. In section 3 we introduce sobering arcs, prove that an 
open book with a sobering arc is overtwisted, and give some examples. In section 4 we investigate Hopf-bands and open books which have a Hopf-band as a Murasugi summand. In section 5 we show that every overtwisted open book is stably-equivalent to one with a sobering arc. In section [6 we look at contact manifolds that arise as the boundaries of symplectic configurations and show that some of these are overtwisted.

This results of this paper were contained in the author's Ph.D. dissertation [13.

We would like to thank John Etnyre for a great deal of encouragement and many helpful conversations, and the anonymous reviewer for very useful comments.

\section{Background}

Throughout this section we will omit proofs that can be easily found in the literature. In particular, there are now many good references on contact geometry, see [5].

\subsection{Topological conventions}

Unless we explicitly state otherwise all manifolds are three dimensional and oriented, and all surfaces are also oriented.

If $S \subset M$ is a surface with boundary embedded in three-manifold $M$, and $T$ another surface so that $\partial S \subset T$, then the framing difference for $\partial S$ between $S$ and $T$, written $\operatorname{Fr}(\partial S ; S, T)$ or just $\operatorname{Fr}(S, T)$, is the oriented number of intersections between $S$ and a push-off of $\partial S$ along $T$.

An automorphism of a surface is a bijective self-homeomorphism, fixing the boundary point-wise. The mapping torus of a surface automorphism, $\phi: \Sigma \rightarrow$ $\Sigma$, is the three-manifold $\Sigma \times_{\phi} S^{1}=(\Sigma \times I) /(p, 1) \sim(\phi(p), 0)$.

Given a simple closed curve $c \subset \Sigma$ we can define an automorphism $D_{c}^{+}: \Sigma \rightarrow \Sigma$, which has support only near $c$, as follows. Let $N$ be a neighborhood of $c$ which is identified (by oriented coordinate charts) with the annulus $\{a \in \mathbb{C} \mid 1 \leq\|a\| \leq$ $2\}$ in $\mathbb{C}$. Then $D_{c}$ is the map $a \mapsto e^{-i 2 \pi(\|a\|-1)} a$ on $N$, and the identity on $\Sigma \backslash N$. We call $D_{c}^{+}$a positive Dehn twist; the inverse, written $D_{c}^{-}$is a negative Dehn twist. 


\subsection{Open books}

Open books $\left(\Sigma_{i}, \phi_{i}\right), i=1,2$, are equivalent if $\Sigma_{1}=\Sigma_{2}$ and $\phi_{1}$ is isotopic to

$h^{-1} \circ \phi_{2} \circ h$ for some automorphism $h$.

Associated to each open book there is a three-manifold, $M_{\Sigma, \phi}=\left(\Sigma \times_{\phi} S^{1}\right) \cup_{\partial}$ $\left(D^{2} \times \partial \Sigma\right)$, where the union is taken by gluing boundary tori in such a way that the curve $\{p\} \times S^{1}$, for $p \in \partial \Sigma$, glues to meridian $\partial\left(D^{2} \times\{p\}\right)$, and $\partial \Sigma \times\{\theta\}$ glues to $\left\{e^{i \theta}\right\} \times \partial \Sigma$. The link $\{0\} \times \partial \Sigma \subset D^{2} \times \partial \Sigma \subset M_{\Sigma, \phi}$ is called the binding, sometimes we'll write it $\partial \Sigma \subset M_{\Sigma, \phi}$. As expected, equivalent open books give homeomorphic $\left(M_{\Sigma, \phi}, \partial \Sigma\right)$.

Given two open books, and some attaching data, we can get a new open book via the Murasugi sum. Unless stated otherwise our Murasugi sum will be the simplest kind, defined below in terms of rectangles. A more general Murasugi sum, also called generalized plumbing or $*$-product in the literature, can be defined similarly by using polygons with $2 m$ sides.

Definition 2.1 Let $\left(\Sigma_{i}, \phi_{i}\right), i=1,2$, be open books, $l_{i} \subset \Sigma_{i}$ properly embedded arcs, and $R_{i}$ rectangular neighborhoods of $l_{i}$ such that $R_{i} \cap \partial \Sigma_{i}$ is two arcs of $\partial R_{i}$. The surface $\Sigma_{1} * \Sigma_{2}$ is the union $\Sigma_{1} \cup_{R_{i}} \Sigma_{2}$ with $R_{1}$ identified to $R_{2}$ in such a way that $\partial R_{1} \cap \partial \Sigma_{1}=\partial R_{2} \backslash \partial \Sigma_{2}$, and vice versa. (This is the identification which gives a new surface, rather than a branched surface.) The Murasugi sum of the $\left(\Sigma_{i}, \phi_{i}\right)$ along $l_{i}$ is the open book $\left(\Sigma_{1}, \phi_{1}\right) *\left(\Sigma_{2}, \phi_{2}\right)=$ $\left(\Sigma_{1} * \Sigma_{2}, \phi_{1} \circ \phi_{2}\right.$ ) (where each $\phi_{i}$ is understood to extend to $\Sigma_{1} * \Sigma_{2}$ by the identity on $\left.\left(\Sigma_{1} * \Sigma_{2}\right) \backslash \Sigma_{i}\right)$. We will often suppress mention of the attaching $\operatorname{arcs} l_{i}$ when they are clear or irrelevant.

Theorem 2.2 The manifold $M_{\left(\Sigma_{1}, \phi_{1}\right) *\left(\Sigma_{2}, \phi_{2}\right)}$ is $M_{\Sigma_{1}, \phi_{1}} \# M_{\Sigma_{2}, \phi_{2}}$.

Proof See 8 .

The Hopf-bands, $H^{ \pm}$, are the open books with surface an annulus and monodromy a single positive or negative Dehn twist about the center of the annulus.

\subsection{Contact structures}

A one dimensional submanifold $L \subset(M, \xi)$ is Legendrian if $T_{p} L \subset \xi$ at each $p \in L$, and is transverse if $T_{p} L \not \subset \xi$. Any curve in a contact manifold can be made Legendrian or transverse by a $C^{0}$-small perturbation. 
Given an embedded surface $S \subset(M, \xi)$ the contact planes restrict to a (singular) line field on $S$. This singular line field integrates into a singular foliation (since any line field is integrable), which we call the characteristic foliation, $\left.\xi\right|_{S}$.

For $S \subset M^{3}$ a surface with $\partial S$ Legendrian, $t w(\partial S, S)$ is the difference in framing of $\partial S$ between $\xi$ and $S$. (That is the intersection number of $S$ with a push-off of $\partial S$ along $\xi$.)

A contact structure is overtwisted if there is an embedded disk $D$ with Legendrian boundary such that $t w(\partial D, D)=0$. A contact structure that is not overtwisted is tight.

We now state some key theorems about overtwisted contact structures:

Theorem 2.3 (The Bennequin Inequality) If $(M, \xi)$ is a tight contact manifold, $S \subset M$ is an embedded surface, and $\partial S$ is Legendrian, then:

$$
t w(\partial S, S) \leq-\chi(S)-|r| \leq-\chi(S)
$$

In the previous theorem, $r$ is the rotation number of the contact structure over the surface. We will use only the courser inequality in this paper.

Theorem 2.4 (Eliashberg [3]) Overtwisted contact structures are isotopic if, and only if, they are homotopic as plane fields.

The following theorem is due to Lutz and Martinet (see [3] for a review):

Theorem 2.5 There is an overtwisted contact structure in each homotopy class of plane fields.

A surface $S \subset(M, \xi)$ is convex if there is a vector field transverse to $S$ whose flow preserves $\xi$. If $v$ is such a vector field, the curves $\Gamma=\{p \in S \mid v(p) \in \xi\}$ are called the dividing curves (or divides) of $S$. For a convex surface with Legendrian boundary $t w(\partial S, S)$ is negative half the number of intersection points in $\Gamma \cap \partial S$.

In [10] Giroux gives a useful characterization of convex surfaces. A characteristic foliation with isolated singularities is called Morse-Smale if all singularities are hyperbolic (in the dynamical systems sense) and there are no saddle-to-saddle connections. (There is another technical requirement in the definition of MorseSmale, it will be satisfied if there are no closed orbits, and this will be enough for us.) A surface $S \subset(M, \xi)$ is convex if and only if $\left.\xi\right|_{S}$ is Morse-Smale. For details see [10. 
Lemma 2.6 Any closed surface $S \subset(M, \xi)$ can be made convex by a $C^{0}$-small perturbation. A surface with Legendrian boundary can be made convex by a $C^{0}$-small perturbation, relative to $\partial S$, if $t w(\partial S, S) \leq-1$ for each component of $\partial S$.

We'll frequently need the following lemma, called the Legendrian Realization Principal (LeRP), due to Ko Honda [14]:

Lemma 2.7 (LeRP) Let $c \subset S$ be a set of curves and arcs (possibly with $\partial c \subset \partial S)$, on a convex surface $S$. If each component of $S \backslash c$ intersects the dividing curves of $S$, then $S$ may be perturbed, by a $C^{0}$-small perturbation through convex surfaces, to make $c$ Legendrian.

We will often use "LeRP" as a verb, meaning "to apply lemma 2.7 to a set of curves".

The folding trick, implicit in [14:

Lemma 2.8 Let $c \subset S$ be a non-separating collection of simple closed curves on convex surface $S$ which don't intersect the divides $\Gamma \subset S$, and $c^{\prime}$ a parallel copy of $c$. Then $S$ can be perturbed to a convex surface divided by $\Gamma \cup c \cup c^{\prime}$. This perturbation is $C^{0}$-small, but not through convex surfaces.

\subsection{Compatibility}

As mentioned above, an open book is compatible with a contact structure, $\xi$ if there is a homotopy $\xi_{t}$ of plane fields (smooth for $t>0$ ) so that $\xi_{1}=\xi, \xi_{0}$ is tangent to the pages, $\xi_{t}$ is contact for $t>0$, and the binding is transverse to $\xi_{t}$.

Torisu shows in [17 that Murasugi sum corresponds to contact connect sum for the compatible contact structure, that is $\xi_{B * C}=\xi_{B} \# \xi_{C}$ (where equality means contact isotopy). Since $H^{+}$is compatible with the standard structure $\xi_{0}$, this implies (see [1]) that $\xi_{B * H^{+}}=\xi_{B}$. We say that $B * H^{+}$is a stabilization of $B$ and that two open books related by a series of stabilizations are stablyequivalent.

A key theorem of Giroux relates compatible open books ([1], see [13] for an exposition of the proof):

Theorem 2.9 (Giroux) Two open books compatible with a fixed contact structure are stably-equivalent. 


\section{$3 \quad$ Sobering arcs}

Definition 3.1 Let $\alpha, \beta \subset \Sigma$ be properly embedded oriented arcs which intersect transversely, $\Sigma$ an oriented surface. If $p \in \alpha \cap \beta$ and $T_{\alpha}, T_{\beta}$ are tangent vectors to $\alpha$ and $\beta$ (respectively) at $p$, then the intersection number $i_{p}=+1$ if the ordered basis $\left(T_{\alpha}, T_{\beta}\right)$ agrees with the orientation of $\Sigma$ at $p$, and $i_{p}=-1$ otherwise. Let $\tilde{\beta}$ be an arc which minimizes intersections with $\alpha$ over boundary fixing isotopies of $\beta$. Sign conventions are illustrated in Figure 1. We define the following intersection numbers:

(1) The algebraic intersection number, $i_{\text {alg }}(\alpha, \beta)=\sum_{p \in \alpha \cap \beta \cap i n t(\Sigma)} i_{p}$, is the oriented sum over intersections on the interior of $\Sigma$.

(2) The geometric intersection number, $i_{\text {geom }}(\alpha, \beta)=\sum_{p \in \alpha \cap \tilde{\beta} \cap i n t(\Sigma)}\left|i_{p}\right|$, is the unsigned count of interior intersections, minimized over all boundary fixing isotopies.

(3) The boundary intersection number, $i_{\partial}(\alpha, \beta)=\frac{1}{2} \sum_{p \in \alpha \cap \tilde{\beta} \cap \partial \Sigma} i_{p}$, is onehalf the oriented sum over intersections at the boundaries of the arcs, after the arcs have been isotoped, fixing boundary, to minimize geometric intersection.

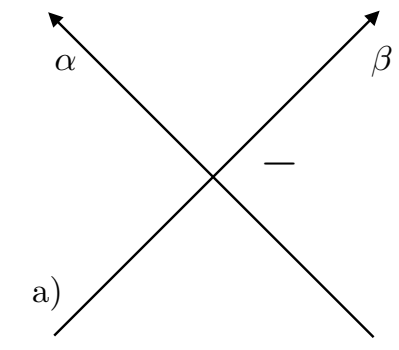

b)
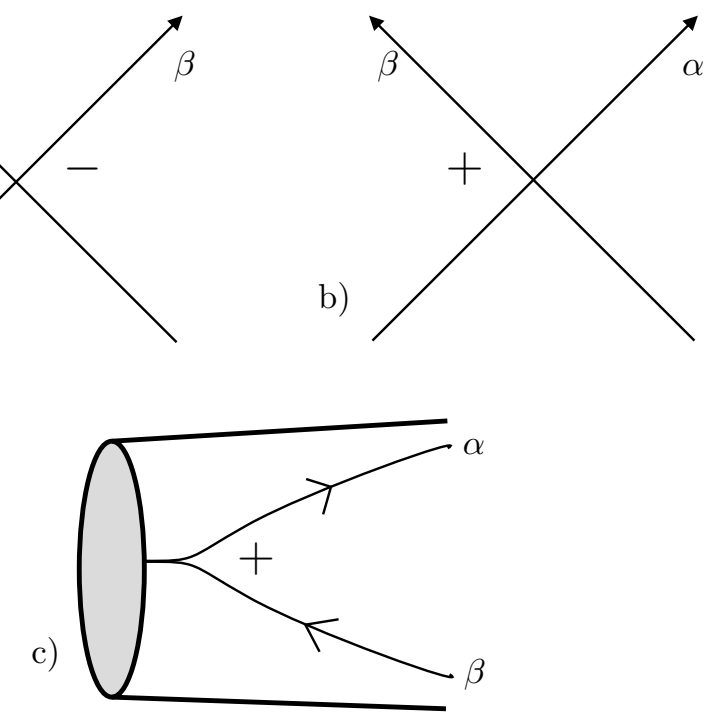

Figure 1: The sign conventions for the intersection of curves $(\alpha, \beta)$ : a) negative intersection, b) positive, c) a positive intersection as it appears at the boundary of the surface 
In particular, for an arc $\alpha \subset \Sigma$ in the page of an open book $(\Sigma, \phi)$ we may consider the intersection numbers: $i_{\text {alg }}(\alpha, \phi(\alpha)), i_{\text {geom }}(\alpha, \phi(\alpha)), i_{\partial}(\alpha, \phi(\alpha))$. We orient $\phi(\alpha)$ by reversing a pushed forward orientation on $\alpha$. Since reversing the orientation of $\alpha$ will also reverse that on $\phi(\alpha)$, without changing the intersection signs, it is irrelevant which orientation we choose.

Much subtle information about a surface automorphism can be gleaned by comparing these intersection numbers for properly embedded arcs. For instance the algebraic monodromy (the induced map $\phi_{*}: H_{*}(\Sigma) \mapsto H_{*}(\Sigma)$,) is unable to distinguish between $(\Sigma, \phi)$ and $\left(\Sigma, \phi^{-1}\right)$, since $\phi_{*}$ is related to $\phi_{*}^{-1}$ by conjugation (through the change of basis which reverses the orientation of each basis vector). However, $i_{\partial}(\alpha, \phi(\alpha))=-i_{\partial}\left(\alpha, \phi^{-1}(\alpha)\right)$ is a geometrically well-defined quantity which can make this distinction.

Definition 3.2 A properly embedded arc $\alpha \subset \Sigma$ is sobering, for a monodromy $\phi$, if $i_{\text {alg }}(\alpha, \phi(\alpha))+i_{\partial}(\alpha, \phi(\alpha))+i_{\text {geom }}(\alpha, \phi(\alpha)) \leq 0$, and $\alpha$ is not isotopic to $\phi(\alpha)$.

In particular, since $i_{\partial} \geq-1$ and each positive intersection contributes 2 to the sum of intersection numbers, there can be no interior intersections with positive sign. So we can reinterpret the definition:

Lemma 3.3 An arc, $\alpha$, is sobering if and only if, after minimizing geometric intersections, $i_{\partial} \leq 0$, there are no positive (internal) intersections of $\alpha$ with $\phi(\alpha)$, and $\alpha$ isn't isotopic to $\phi(\alpha)$.

There is a good reason to be interested in such arcs:

Theorem 3.4 If there is a sobering arc $\alpha \subset \Sigma$ for $\phi$, then the open book $(\Sigma, \phi)$ is overtwisted.

To prove this theorem we will construct a surface with Legendrian boundary which violates the Bennequin inequality, theorem 2.3. Since this inequality is a necessary condition for tightness, we'll conclude that $\xi_{\Sigma, \phi}$ is overtwisted. In doing so, however, we will not find an overtwisted disk - such a disk must exist, but will, in general, not be nicely positioned with respect to the open book.

We begin our construction by suspending $\alpha$ in the mapping torus: $\alpha \times I \subset$ $\Sigma \times{ }_{\phi} I$. Let $p \in \partial \Sigma$ be an endpoint of $\alpha$. Since $\phi$ fixes the boundary, $p \times I$ is a meridian around the binding. Let $D_{\alpha}$ be the surface made by gluing a meridional disc to $\alpha \times I \subset M_{\Sigma, \phi}$ along $p \times I$ for each $p \in \partial \alpha$ (this extends 
$\alpha \times I$ across the binding in $\left.M_{\Sigma, \phi}\right)$. The surface $D_{\alpha}$ is a disk which has $\partial D_{\alpha} \subset \Sigma$, and is embedded on its interior, but possibly immersed on its boundary.

We isotope $\phi(\alpha)$, relative to the boundary, to have minimal and transverse intersection with $\alpha$. This induces an isotopy of $D_{\alpha}$, which intersects itself at transverse double points on its boundary. To proceed we need to remove the double points of $D_{\alpha}$. However, if we simply separate the edges of the disk meeting at an intersection, the boundary of the disk won't stay on $\Sigma$, eliminating any control we might have had over the framing. Instead we will opt to resolve the double points by decreasing the Euler characteristic of the surface:

Definition 3.5 Let $F$ be an orientable surface which is embedded on $\operatorname{Int}(F)$ and has isolated double points on $\partial F$. The resolution of $F$ is constructed by thickening each double point into a half-twisted band in such a way that the resulting surface remains oriented. This is done according to the following local model (see Figure 2). In $\mathbb{R}^{3}$ coordinates, in a small neighborhood of a double point, $F$ looks like $\left\{(x, y, z) \in \mathbb{R}^{3} \mid z \geq 0, y=0\right\} \cup\left\{(x, y, z) \in \mathbb{R}^{3} \mid z \leq 0, x=0\right\}$, oriented by $\hat{x}$ and $\pm \hat{y}$, respectively. We resolve by gluing in the triangles $\left\{(x, y, z) \in \mathbb{R}^{3} \mid z=0, x \geq 0,0 \geq \pm y \geq x-1\right\}$ and $\left\{(x, y, z) \in \mathbb{R}^{3} \mid z=0, x \leq\right.$ $0,0 \leq \pm y \leq x+1\}$ (that is, a pair of triangles depending on the intersection sign). Note that the boundary of both $F$ and its resolution are on the plane $z=0$.

Let $S$ be the resolution of $D_{\alpha}$, with additionally $\partial D_{\alpha}$ pushed into $\operatorname{Int}(\Sigma)$ at the points $\partial \alpha$ (see Figure 3). Figure 2 $\mathrm{b}$ ) shows, on $\Sigma$, how the boundary $\partial D_{\alpha}$ changes into $\partial S \subset \Sigma$ at a resolution.

Lemma 3.6 $\chi(S)=1-i_{\text {geom }}(\alpha, \phi(\alpha))$.

Proof The construction of $S$ starts with the disk $D_{\alpha}$ which has $i_{\text {geom }}(\alpha, \phi(\alpha))$ double points on its boundary. At each double point we glue a band from the disk to itself. The counting follows since a disk has $\chi=1$, and adding a band reduces the Euler characteristic by 1 .

Lemma 3.7 The difference in framings of $\partial S$ with respect to $S$ and $\Sigma$ is: $\operatorname{Fr}(\partial S ; S, \Sigma)=-i_{\text {alg }}(\alpha, \phi(\alpha))-i_{\partial}(\alpha, \phi(\alpha))$.

Proof The relative framing can be computed as the oriented intersection number of $S$ with a push-off of $\partial S$ along $\Sigma$. To choose the push-off first choose 

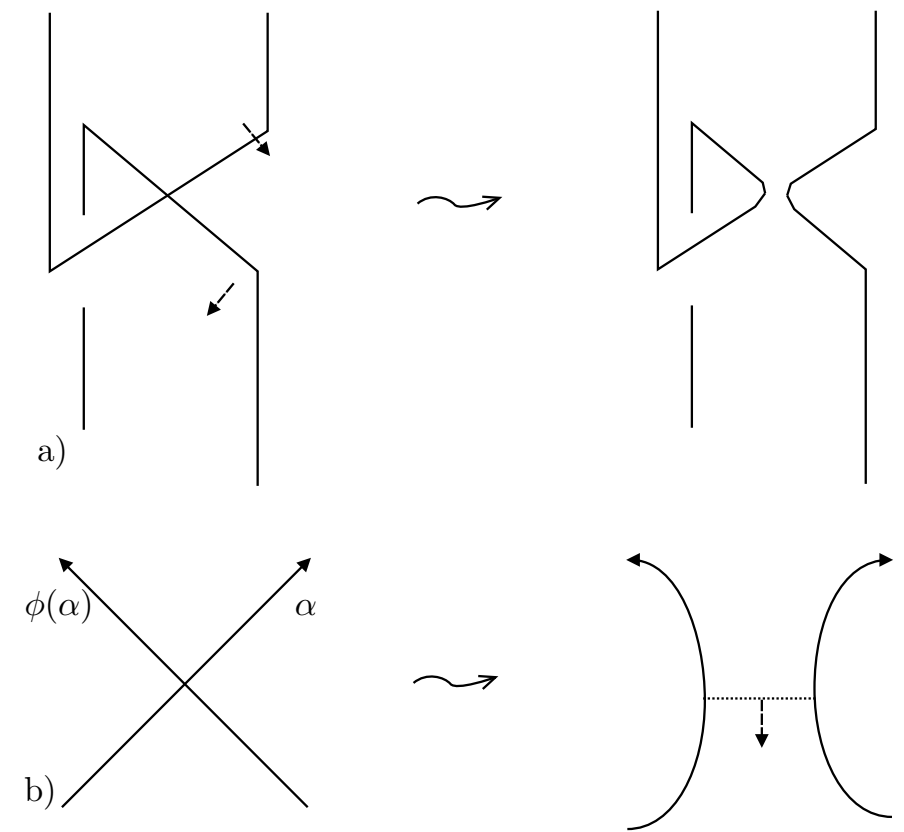

Figure 2: The local model for resolutions of immersed points on the boundary of a surface: a) the surface, with dashed arrows indicating orientations, b) the resolution of $\alpha \cap \phi(\alpha)$, drawn on $\Sigma$, for the case of $D_{\alpha}$ (the dotted line represents $\left.\operatorname{Int}\left(D_{\alpha}\right) \cap \Sigma\right)$
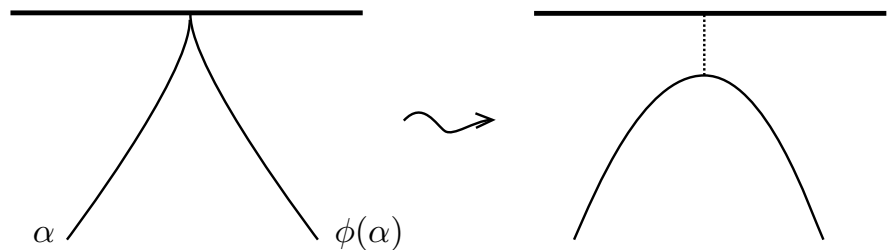

Figure 3: Smoothing the boundary of the suspension disk, $D_{\alpha}$ : The dashed line is $D_{\alpha} \cap \Sigma$.

an orientation for $S$, and let $s$ be a tangent vector to $\partial S$ which gives the boundary orientation. Choose vector $t \in T \Sigma$ so that $(s, t)$ provide an oriented basis agreeing with the orientation of $\Sigma$. Let $L$ be the push-off of $\partial S$ in the $t$ direction.

Now, $L \subset \Sigma$ can intersect $S$ only on $\operatorname{Int}(S) \cap \Sigma$. This set consists of arcs where intersections were resolved in the construction of $S$ (including the boundary intersections, which are smoothed, as in Figure 3). For each of the internal 
intersections of $\alpha$ with $\phi(\alpha), L$ intersects $S$ once with sign opposite that of the intersection, as shown in Figure 4.

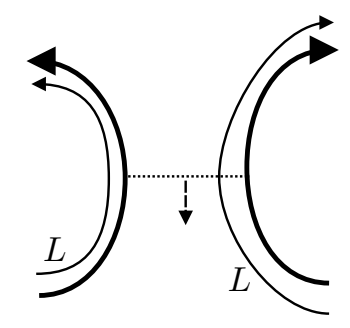

Figure 4: Framing at the resolution of a positive intersection: The dashed line is $S \cap \Sigma$, while the arrow is the orientation of $S . L$ is a push-off of $\partial S$.

It remains to account for the boundary intersections. Since $\phi$ fixes $\partial \Sigma$ (as for any open book), there are exactly two intersections points in $\partial \alpha \cap \partial \phi(\alpha)=\partial \alpha$ (so $i_{\partial}(\alpha, \phi(\alpha)) \in\{+1,-1,0\}$ ). There are four possibilities for the intersection of $L$ with $S$ at these resolutions, according to the signs of the intersections at the beginning and end of $\alpha$. One can compute each of these possibilities, as in the example of Figure 5 the result is shown in table 11. There will be one intersection near the boundary, with sign $\mp 1$, if $i_{\partial}(\alpha, \phi(\alpha))= \pm 1$, and either two with opposite signs, or none, if $i_{\partial}(\alpha, \phi(\alpha))=0$. Therefore the boundary intersections contribute $-i_{\partial}(\alpha, \phi(\alpha))$ to the framing, $\operatorname{Fr}(S, \Sigma)$.

Finally, in the above analysis we chose an orientation on $\alpha$ (which then orients $\phi(\alpha), D_{\alpha}$ and $\left.S\right)$. Reversing this orientation is equivalent to reversing the orientation of $\partial S$, and the effect on the framing computation is to take the push-off of $\partial S$ along $\Sigma$ but to the other side of $S$. However, the relative framing $\operatorname{Fr}(S, \Sigma)$ is well-defined independent of which push-off is used, so our result doesn't depend on the chosen orientation. (The independence can also be seen directly by repeating the calculation using the other push-off.)

\begin{tabular}{|l|l|l|}
\hline & start $\alpha$ & end $\alpha$ \\
\hline pos. & -1 & 0 \\
neg. & 0 & +1 \\
\hline
\end{tabular}

Table 1: The framing intersections contributed by a boundary intersection of the given sign at the beginning or end of $\alpha$. Computations were made as in Figure 5

We are now ready to prove the main theorem of this section. 


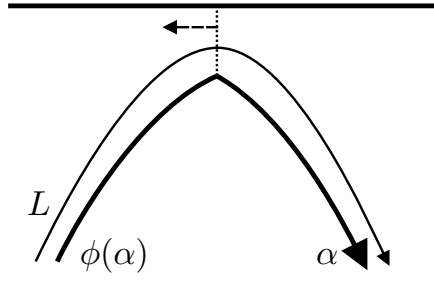

Figure 5: Framing near boundary: an example of computing the intersection of $L$ with $S$, on $\Sigma$. Here a positive boundary intersection at the start of $\alpha$ contributes -1 to the framing. The dotted line is $S \cap \Sigma$, while the dashed arrow is the orientation of $S$.

Proof of Theorem 3.4 As we will later wish to LeRP $\partial S$, we must first rule out any components of $\partial S$ which bound disks on $\Sigma$.

Assume there is a disk $D \subset \Sigma$ such that $\partial D \subset \partial S$. The intersection signs at the corners of a disk (where resolutions have occurred on $\partial S$ ) must alternate: see Figure 6 . However, by the sobering condition, there is at most one positive intersection, which occurs at the boundary. This implies that the only possible disk is a bi-gon with a corner on the boundary, but in this case either the intersection $\alpha \cap \phi(\alpha)$ isn't minimal, or the other corner of the bi-gon is also on the boundary. The former contradicts the minimal set-up we've arranged, the latter contradicts the definition ( $\alpha$ isn't isotopic to $\phi(\alpha)$ ), so there can be no such disk.

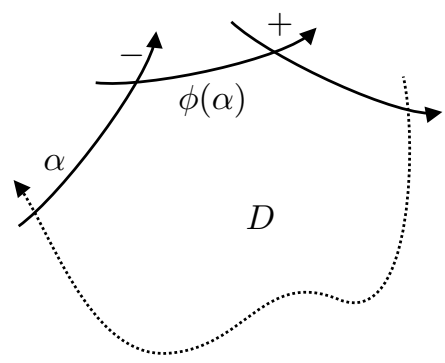

Figure 6: If disk $D \subset \Sigma$ was created by resolving $\alpha \cap \phi(\alpha)$, the signs at the intersections would have to alternate.

In order to use the machinery of convex surfaces, we must make the leaves convex. We can do this by an isotopy of the contact structure.

Lemma 3.8 Let $(\Sigma, \phi)$ be an open book, $\pi: M_{\Sigma, \phi} \backslash \partial \Sigma \rightarrow S^{1}$ the fibration of the complement to the binding. The closure of $\pi^{-1}(0) \cup \pi^{-1}(0.5)$ is convex and 
divided by the binding, $\partial \Sigma=\partial\left(\pi^{-1}(0)\right)$, for some compatible contact structure.

Proof Let $\tilde{\Sigma}$ be the closure of $\pi^{-1}(0) \cup \pi^{-1}(0.5)$ (oriented by $\pi^{*}(d s)$ on $\pi^{-1}(0)$ and $\pi^{*}(-d s)$ on $\left.\pi^{-1}(0.5)\right)$, we take a compatible contact structure built as in [16] from a one-form $\lambda$ (see [13]). We may choose the homotopy extending $\lambda$ to the mapping torus in such a way that the characteristic foliation on $\tilde{\Sigma}$ looks like $\operatorname{ker}\left(\lambda+\phi^{*}(\lambda)\right)$ on $\pi^{-1}(0.5)$ (and like $\operatorname{ker}(\lambda)$ on $\pi^{-1}(0)$ ). By a small perturbation of this homotopy (eg. by perturbing $\phi$ ) we can assure that the saddles on $\pi^{-1}(0)$ connect to the sources/sinks on $\pi^{-1}(0.5)$, and vice versa, hence the characteristic foliation is Morse-Smale. By the results of [10], a surface with such a characteristic foliation is convex. The dividing curves separate the regions where the contact one-form is positive on a positive normal vector from those where it is negative. Since the contact structure is compatible, $\pi^{-1}(0)$ is positive while $\pi^{-1}(0.5)$ is negative (with the natural orientation: $\pi^{*}(d s)$, on each), hence the dividing curve is exactly the binding.

Because the compatible contact structure to an open book is unique up to isotopy, we can perform an isotopy to assure that our contact structure is that of the lemma. Now, with convex pages, we want to apply the LeRP (lemma 2.7) to the curves $\partial S$, but we must insure that there is a component of the dividing set in each component of $\tilde{\Sigma} \backslash \partial S$. To achieve this, let $\Gamma$ contain a simple closed curve in each component of $\tilde{\Sigma} \backslash \partial S$. Using the folding lemma 2.8 along $\Gamma$ we perturb $\tilde{\Sigma}$, changing the dividing set to $\partial \Sigma \cup \Gamma \cup \Gamma^{\prime}\left(\Gamma^{\prime}\right.$ a parallel copy of $\Gamma$ ). Now we can apply the LeRP, perturbing $\tilde{\Sigma}$ again to make the curves $\partial S$ Legendrian.

By the definition of convex surfaces there is a contact vector field $v$ normal to $\tilde{\Sigma}$. Since $\partial S$ doesn't cross the dividing set, $\left.v\right|_{\partial S} \notin \xi_{\Sigma, \phi}$, so the framing of $S$ with respect to $\xi_{\Sigma, \phi}$ (the twisting number) may be computed by using a push-off of $\partial S$ in the $v$ direction. But $v$ is normal to $\tilde{\Sigma}$ so a push-off along $\tilde{\Sigma}$ is equivalent, for framing purposes, to the push off along $v$. Hence, the framing coming from $\xi_{\Sigma, \phi}$ is the same as that from $\Sigma: t w(\partial S, S)=\operatorname{Fr}(\partial S ; S, \Sigma)$.

We computed above that $\operatorname{Fr}(S, \Sigma)=-i_{\text {alg }}(\alpha, \phi(\alpha))-i_{\partial}(\alpha, \phi(\alpha))$. So,

$$
t w(\partial S, S)+\chi(S) \geq-i_{a l g}(\alpha, \phi(\alpha))-i_{\partial}\left(\alpha, \phi(\alpha)+1-i_{\text {geom }}(\alpha, \phi(\alpha)) .\right.
$$

The sobering condition $-i_{\text {alg }}(\alpha, \phi(\alpha))-i_{\partial}\left(\alpha, \phi(\alpha)-i_{\text {geom }}(\alpha, \phi(\alpha)) \geq 0\right.$ then implies:

$$
t b(\partial S, S)+\chi(S)>0,
$$

violating the Bennequin inequality (theorem 2.3), which must hold if $\xi_{\Sigma, \phi}$ is tight. Hence, $(\Sigma, \phi)$ is overtwisted. 
Remark A word about the use of arcs instead of closed curves inside $\Sigma$. Our construction, above, would fail for these curves for simple Euler characteristic reasons, or we may rule out such considerations more directly: in the complement of the binding $\xi_{\Sigma, \phi}$ is a perturbation of the taught foliation given by the leaves of the open book. From the results of Eliashberg and Thurston [4] such a contact structure is symplectically fillable, and hence tight. Thus, no surface entirely in the complement of the binding, such as the suspension of a closed curve on $\Sigma$, can violate the Bennequin inequality.

We can slightly extend the class of monodromies for which sobering arcs are useful by a little cleverness:

Theorem 3.9 If an arc $\alpha \subset \Sigma$ has:

$$
i_{a l g}(\alpha, \phi(\alpha))+i_{\partial}(\alpha, \phi(\alpha))+i_{g e o m}(\alpha, \phi(\alpha))=-1,
$$

then each open book $\left(\Sigma, \phi^{n}\right)$, for $n>0$, is overtwisted.

Proof The open book $\left(\Sigma, \phi^{n}\right)$ can be built by gluing $n$-copies of $\Sigma \times I$ to each other by $\phi$ (including the bottom of the first to the top of the last) to get the mapping torus $\Sigma \times_{\phi^{n}} S^{1}$, then adding the binding as usual. In each $\Sigma \times I$ we have a disk $\alpha \times I$. The top of one disk intersects the bottom of the next as $\alpha \cap \phi(\alpha)$. If we resolve these intersections, as in the previous proofs, we get a surface with $\chi=n-n i_{\text {geom }}(\alpha, \phi(\alpha))$ and framing $n i_{a l g}(\alpha, \phi(\alpha))$. Next we add in the binding and cap off the surface with disks $D^{2} \times \partial \alpha$, to get a surface $S$ with $\chi(S)=2-n-n i_{\text {geom }}(\alpha, \phi(\alpha))$ and framing $n i_{\partial}(\alpha, \phi(\alpha))+n i_{a l g}(\alpha, \phi(\alpha))$.

To finish the proof we proceed exactly as in the proof of theorem 3.4 rule out components of $\partial S$ which bound disks on a page, then fold and LeRP to make $\partial S$ Legendrian (we'll have to do this along several pages, but they won't interfere since folding and LeRPing can be done in small neighborhoods). Finally we get a surface with $t b(\partial S, S)+\chi(S)=-n\left(i_{a l g}(\alpha, \phi(\alpha))+i_{\partial}(\alpha, \phi(\alpha))\right)+2-n$. Then under the condition $i_{\text {alg }}(\alpha, \phi(\alpha))+i_{\partial}(\alpha, \phi(\alpha))+i_{\text {geom }}(\alpha, \phi(\alpha))=-1$ we get: $t b(\partial S, S)+\chi(S)=n+2-n=2>0$, so the open book is overtwisted.

We can apply this theorem to get some interesting examples:

Corollary 3.10 Let $\Sigma_{g}$ be a surface with genus $g>0$ and one boundary component, and let $\delta \subset \Sigma_{g}$ be parallel to the boundary. The open book $\left(\Sigma_{g}, D_{\delta}^{-n}\right)$ is overtwisted $(n>0)$. 
Proof Let $\left\{a_{i}, b_{i}\right\}_{i=1, \ldots, g}$ be simple closed curves on $\Sigma_{g}$ such that $a_{i}$ intersects $b_{i}$ in one point, $b_{i}$ intersects $a_{i+1}$ in one point, and the curves are otherwise disjoint. One can show, as in [15], that $D_{\delta}=\left(\prod_{i} D_{a_{i}} D_{b_{i}}\right)^{4 g+2}$.

Then $D_{\delta}^{-n}=\left(D_{b_{g}}^{-} D_{a_{g}}^{-} \cdots D_{b_{1}}^{-} D_{a_{1}}^{-}\right)^{n(4 g+2)}$. An arc which intersects only $b_{g}$ will be sobering for $\phi=D_{b_{g}}^{-} D_{a_{g}}^{-} \cdots D_{b_{1}}^{-} D_{a_{1}}^{-}$.

On the other hand, since the open books $\left(\Sigma_{g}, D_{\delta}^{n}\right)$ are positive, they are Stein fillable, and hence tight. (The connection between fillable contact structures and positive monodromy was found by many people, see [7] for a review.)

We return now to the Poincare homology-sphere with reversed orientation, discussed in the introduction. This manifold arises as +1 -surgery on the trefoil $T^{+} \subset S^{3}$, so it supports an open book which comes from the natural fibering of $S^{3} \backslash T^{+}$by adding a boundary parallel negative Dehn twist. (To see this, push the surgery curve onto a page, then verify directly that an arc passing the surgery experiences a negative Dehn twist). Explicitly: let $\Sigma$ be the punctured torus, $\delta$ a boundary parallel curve, and $a, b$ curves which intersect in a point. Then the open book $\left(\Sigma, D_{\delta}^{-1} D_{b}^{+1} D_{a}^{+1}\right)$ represents the Poincare homology-sphere with reversed orientation. The technique of the corollary shows that the monodromy can be written $\phi=\left(D_{b}^{-} D_{a}^{-}\right)^{6}\left(D_{b}^{+} D_{a}^{+}\right)=\left(D_{b}^{-} D_{a}^{-}\right)^{5}$, and that this is overtwisted.

\section{A special case}

Let us consider the simplest open books. In a disk all arcs are boundary parallel, so we turn our attention to annuli, and especially to the Hopf-bands, $H^{ \pm}$, which are annuli with monodromy a single right or left-handed Dehn twist along, $c$, the center of the annulus. If we let $\alpha$ be an arc which crosses the annulus, we see from Figure 7 that $i_{\text {geom }}\left(\alpha, D_{c}^{ \pm}(\alpha)\right)=i_{\text {alg }}\left(\alpha, D_{c}^{ \pm}(\alpha)\right)=0$, while $i_{\partial}\left(\alpha, D_{c}^{ \pm}(\alpha)\right)= \pm 1$ for $H^{ \pm}$, respectively. Since $D_{c}^{ \pm}(\alpha)$ isn't isotopic to $\alpha, \alpha$ is sobering for $H^{-}$(but not for $H^{+}$!).

Lemma 4.1 The Murasugi sum $(\Sigma, \phi) * H^{-}$, for any open book $(\Sigma, \phi)$, along any attaching curve, is overtwisted.

Proof Take an arc across $H^{-}$, outside the attaching region. It is unmoved by (the extension of) $\phi$, so will have the same intersection properties relative to $D_{c}^{-} \circ \phi$ as the arc $\alpha$, above, had relative to $D_{c}^{-}$. So this arc will be sobering in the sum. 


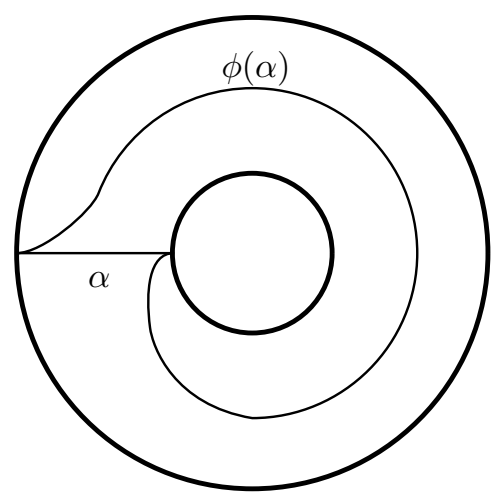

Figure 7: A negative Hopf-band, $H^{-}$, with the transverse arc, $\alpha$, and its image

How special are the intersection properties of the arcs we've just considered? It turns out that they uniquely specify the geometric situation of having a Hopf-band Murasugi summand.

Theorem 4.2 If $i_{\text {geom }}(\alpha, \phi(\alpha))=0$ and $i_{\partial}(\alpha, \phi(\alpha))= \pm 1$, then $\alpha$ is the transverse arc to a positive (respectively negative) Hopf-band. That is $(\Sigma, \phi)=$ $\left(\Sigma^{\prime}, \phi^{\prime}\right) * H^{ \pm}$where $\alpha$ is parallel to the attaching curve in $H^{ \pm}$.

Proof Let $c \subset \Sigma$ be the curve which comes from smoothing $\alpha \cup \phi(\alpha)$. Since $i_{\text {geom }}(\alpha, \phi(\alpha))=0, c$ will be a simple closed curve. Note that $\phi(\alpha)=D_{c}^{ \pm}(\alpha)$ up to isotopy, where the sign of the Dehn-twist agrees with $i_{\partial}(\alpha, \phi(\alpha))$.

Let $\phi^{\prime}=D_{c}^{\mp} \circ \phi$ on the surface $\Sigma^{\prime}=\Sigma \backslash \alpha$. This makes sense since $\phi^{\prime}(\alpha)=$ $D_{c}^{\mp} \circ \phi(\alpha)=D_{c}^{\mp} \circ D_{c}^{ \pm}(\alpha)=\alpha$ (up to isotopy, so for some representative of the isotopy class of $\left.\phi^{\prime}\right)$.

If we plumb a Hopf-band onto $\left(\Sigma^{\prime}, \phi^{\prime}\right)$ along $c \backslash \alpha \subset \Sigma^{\prime}$, we get the map $D_{c}^{ \pm} \circ \phi^{\prime}=\phi$ on $\Sigma$. Thus $(\Sigma, \phi)=\left(\Sigma^{\prime}, \phi^{\prime}\right) * H^{ \pm}$, and $\alpha$ crosses the one-handle, as required.

Of course if $(\Sigma, \phi)=\left(\Sigma^{\prime}, \phi^{\prime}\right) * H^{ \pm}$, then there is such an arc $\alpha$, described above, so we in fact have a necessary and sufficient condition for the existence of a Hopf-band summand in an open book. This criterion can be used to show that certain fibered links are not Hopf plumbings, and it is often helpful in finding stabilizations in otherwise difficult examples, as in section 6 . 


\section{A criterion for overtwisting}

Theorem 5.1 An open book is overtwisted if and only if it is $\mathrm{H}^{+}$-stably equivalent to an open book with a sobering arc.

Proof If: The final open book of the equivalence has a sobering arc so its compatible contact structure is overtwisted. Neither summing nor de-summing $\mathrm{H}^{+}$changes the contact structure, so the original open book is overtwisted.

Only If: For any open book $B$ the homotopy classes of plane fields on $M_{B}$ have a natural $\mathbb{Z}$-action, and we arrive in the homotopy class of $\xi_{B * H^{-}}$by acting on the homotopy class of $\xi_{B}$ with +1 (see [12]).

Now let $(\Sigma, \phi)$ be an overtwisted open book, let $\xi$ be a contact structure in the homotopy class of $\xi_{(\Sigma, \phi)}$ acted on by -1 (there is a contact structure in any homotopy class), and let $B$ be an open book compatible with $\xi$. The contact structure $\xi_{B * H^{-}}$then is homotopic to $\xi_{(\Sigma, \phi)}$, and both are overtwisted, hence they are isotopic (lemma 2.4). So, $B * H^{-}$is $H^{+}$-stably equivalent to $(\Sigma, \phi)$ by theorem [2.9] and $B * H^{-}$has a sobering arc (across the Hopf band, see lemma 4.11).

\section{Boundaries of symplectic configurations}

Following [9] we define a symplectic configuration graph to be a labeled graph $G$ with no edges from a vertex to itself and with each vertex $v_{i}$ labeled with a triple $\left(g_{i}, m_{i}, a_{i}\right)$, where $g_{i} \in\{0,1,2, \ldots\}, m_{i} \in \mathbb{Z}$ and $a_{i} \in(0, \infty)$. ( $a_{i}$ doesn't enter into our purely three-dimensional concerns, so we'll often omit it.) Let $d_{i}$ denote the degree of vertex $v_{i}$. A configuration graph is called positive if $m_{i}+d_{i}>0$ for every vertex $v_{i}$.

Given a positive configuration graph $G$ we define an open book $(\Sigma(G), \phi(G))$ as follows: For each vertex $v_{i}$ let $F_{i}$ be a surface of genus $g_{i}$ with $m_{i}+d_{i}$ boundary components. $\Sigma(G)$ is the surface obtained by connect sum of the $F_{i}$, with one connect sum between $F_{i}$ and $F_{j}$ for each edge connecting $v_{i}$ to $v_{j}$. See Figure 8 for an example. For each edge in $\mathrm{G}$ there is a circle $e_{i j}$ in $\Sigma(G)$. Let $\sigma(G)=\prod D_{e_{i j}}^{+}$, a right-handed Dehn twist at each connect sum. Let $\delta(G)$ be the product of one right-handed Dehn twist around each circle of $\partial \Sigma(G)$. Finally, $\phi(G)=\sigma(G)^{-1} \circ \delta(G)$.

In 9] Gay shows that the open books $(\Sigma(G), \phi(G))$ arise as the concave boundary of certain symplectic manifolds. In that paper he asks the question: 

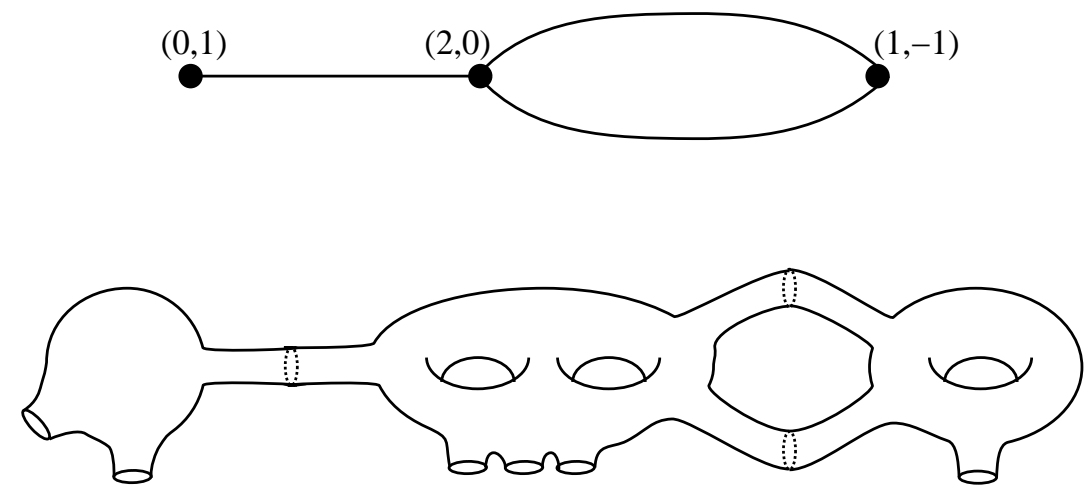

Figure 8: A positive configuration graph (the $a_{i}$ have been suppressed), and the surface $\Sigma(G)$ : The dotted circles are the $e_{i j}$.

Question (Gay 9]) Are there any positive configuration graphs $G$ for which we can show that $(\Sigma(G), \phi(G))$ is overtwisted (and hence conclude that a symplectic configuration with graph $G$ cannot embed in a closed symplectic 4manifold)?

We can use the techniques of the previous sections to give such examples.

First, note that if $l$ is a properly embedded arc in $\Sigma(G)$ which crosses from one $F_{i}$ to an adjacent one, so crosses one negative Dehn twist and two positive, then $i_{\text {geom }}(l, \phi(l))=0$ and $i_{\partial}(l, \phi(l))=+1$ (see Figure 9). From theorem 4.2 we know that there is an $H^{+}$summand. To remove it we'll first re-write the monodromy using the lantern relation (this is a standard fact of mapping class groups, see [2]):

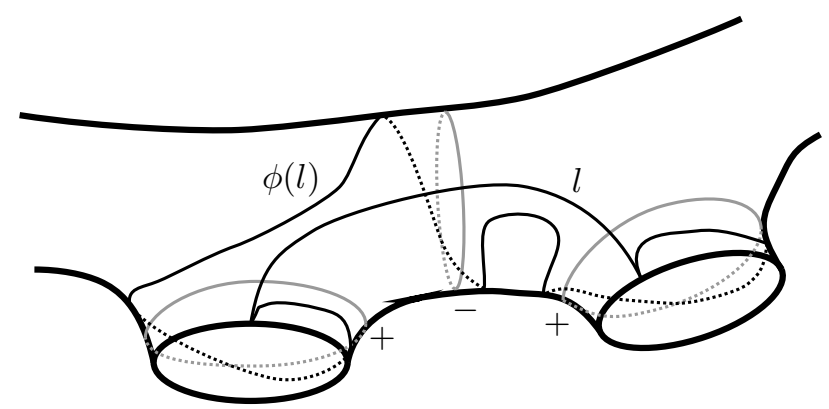

Figure 9: The arc $l \subset \Sigma(G)$, its image under $\phi(G)$, and the twisting curves it crosses 
Lemma 6.1 The following relation holds in the mapping class group of a fourtimes punctured sphere (fixing the boundary point-wise), referring to Figure 10(a) and (b):

$$
D_{a}^{+} \circ D_{b}^{+} \circ D_{c}^{+} \circ D_{d}^{+}=D_{\alpha}^{+} \circ D_{\beta}^{+} \circ D_{\gamma}^{+} .
$$

a)
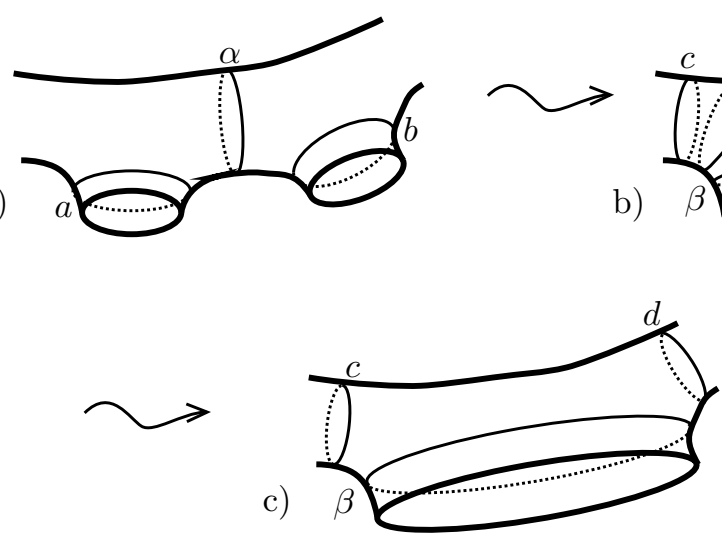

Figure 10: De-plumbing an $H^{+}$from $(\Sigma(G), \phi(G))$. The first step is an application of the lantern lemma to go from $D_{a}^{+} \circ D_{b}^{+} \circ D_{\alpha}^{-}$to $D_{c}^{-} \circ D_{d}^{-} \circ D_{\beta}^{+} \circ D_{\gamma}^{+}$, the second is the removal of a Hopf-band along $\gamma$.

Applying this relation to the region of Figure 9 around arc $l$, we can rewrite the monodromy as in Figure 10 (a) and (b): $D_{a}^{+} \circ D_{b}^{+} \circ D_{\alpha}^{-}=D_{c}^{-} \circ D_{d}^{-} \circ D_{\beta}^{+} \circ D_{\gamma}^{+}$ (Dehn twists about $a, b, c, d$ commute with each other and with $\alpha, \beta$, and $\gamma$, since these pairs don't intersect). It is then clear, since $l$ crosses only the final Dehn twist $D_{\gamma}^{+}$, how to remove a Hopf-band: remove $D_{\gamma}^{+}$and cut along $l$, Figure 10(c). We arrive at a surface with one fewer boundary component, and $D_{c}^{-} \circ D_{d}^{-} \circ D_{\beta}^{+}$replacing $D_{a}^{+} \circ D_{b}^{+} \circ D_{\alpha}^{-}$in the monodromy. We'll use this move repeatedly to prove the next lemma. Note that this move doesn't necessarily return an open book $\left(\Sigma\left(G^{\prime}\right), \phi\left(G^{\prime}\right)\right)$, since the resulting open book may have several negative Dehn twists around the same curve.

Theorem 6.2 Let $G$ be a positive configuration graph with a vertex $v_{1}$ such that $d_{1}=1$ and $g_{1}=0$, but not a graph with:

(1) one edge, $m_{1}=0$,

(2) one edge, $g_{2}=m_{2}=0$,

(3) one edge, $g_{2}=0, m_{2}=m_{1}=1$, or,

(4) two edges, $g_{3}=0, m_{1}=m_{3}=0$. 
Then $(\Sigma(G), \phi(G))$ is overtwisted.

Proof First we apply the move illustrated in Figure 10 repeatedly, removing positive Hopf-bands until all of the boundary components of $F_{1}$ are gone, leaving a boundary component with one positive Dehn twist and $m_{1}+1$ negative twists. These, of course, are equivalent to $m_{1}$ negative Dehn twists. We are left with a surface, $G^{\prime}$, in which $F_{1}$ and $F_{2}$ have been replaced with a surface of genus $g_{2}$, and $m_{2}+d_{2}$ boundary components, which has $m_{1}$ negative Dehn twists about one of these punctures (the remaining monodromy twists are unchanged). See Figure 11.

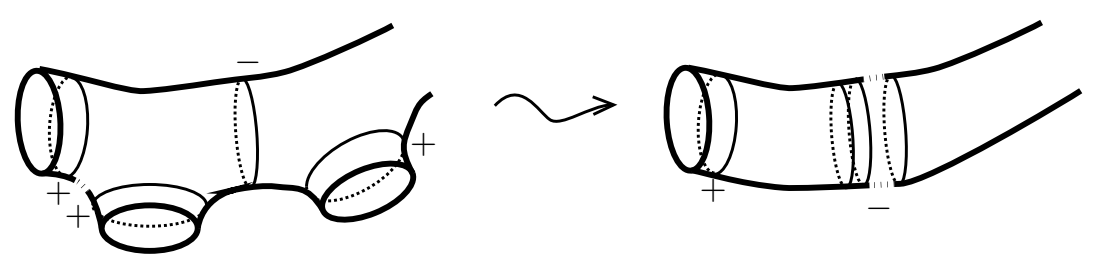

Figure 11: Removing almost all boundary components by applying Figure 10 to remove $H^{+}$

The surface $G^{\prime}$ will have $\sum_{i>1} m_{i}+d_{i}$ boundary components and, since $m_{i}+$ $d_{i}>0$, will have a single boundary component only when $e=1$ and $m_{2}=0$ (where $e$ is the number of edges). In this case we have a surface with one puncture and monodromy consisting of $m_{1}$ negative twists about the boundary; by corollary 3.10 the open book will be overtwisted if $g_{2}>0$ and $m_{1}>0$. The 'bad' cases of $e=1, m_{2}=0$, and either $g_{2}=0$ or $m_{1}=0$ are excluded by hypothesis.

Now if $G^{\prime}$ has several boundary components, let $\alpha$ be an arc from the boundary component with $m_{1}$ negative Dehn twists to another boundary component, chosen so that it crosses as many of the $e_{i j}$ (non-boundary parallel negative twists of $\phi(G))$ as possible, but crosses each at most once. The arc $\alpha$ will cross the $m_{1}$ negative Dehn twists, $k$ additional negative twists (one for each $e_{i j}$ crossed), and finally a positive Dehn twist (see Figure 12). (Note that if $k=0$ then $e=1$, and if $k=1$ then $e=2$ : if there were more edges then $\alpha$ could be extended.)

Before any isotopy there are only negative internal intersections of $\alpha$ with $\phi(\alpha)$, thus after an isotopy which minimizes intersections there will still be only negative internal intersections. The boundary intersection number will initially be 0 (since $\alpha$ crosses only the one positive twist as in Figure 12), and 
can only be reduced as internal intersections are minimized (since all internal intersections are negative). So, $\alpha$ will be sobering if it is not isotopic to $\phi(\alpha)$.

If $m_{1}+k>1$, that is $\alpha$ crosses more than one negative twist, the algebraic intersection number (including boundary intersections) is less than 0 . This is an isotopy invariant, so $\alpha$ is not isotopic to $\phi(\alpha)$.

We can rule out the case $m_{1}+k=0$ since we must have $e=1$ and $m_{1}=0$. What if $m_{1}+k=1$ ? Then $\alpha$ crosses one negative and one positive Dehn twist, call them $D_{\beta}^{+}$and $D_{\gamma}^{-}$. Up to homotopy (relative to the boundary) $[\phi(\alpha)]=[\alpha]+[\beta]+[\gamma]$ (as $\beta$ and $\gamma$ can't cross). If $\alpha$ is isotopic to $\phi(\alpha)$ then $[\beta]=[\gamma]$, up to sign.

There are two cases, first consider $m_{1}=1$ and $k=0$. In this case $e=1$, so both $\beta$ and $\gamma$ are boundary parallel - in order for them to be homotopic $G^{\prime}$ must be a cylinder, so $g_{2}=0$ and $m_{2}=m_{1}=1$ (a case excluded by hypothesis).

In the other case $m_{1}=0$ and $e=2$, now $\beta$ is again boundary parallel (near the boundary of $F_{3}$ ), but $\gamma$ is the curve separating $F_{3}$ from $F_{2}$. Their homotopy equivalence implies that $F_{3}$ is a cylinder, so $g_{3}=0=m_{3}$ (another excluded case).

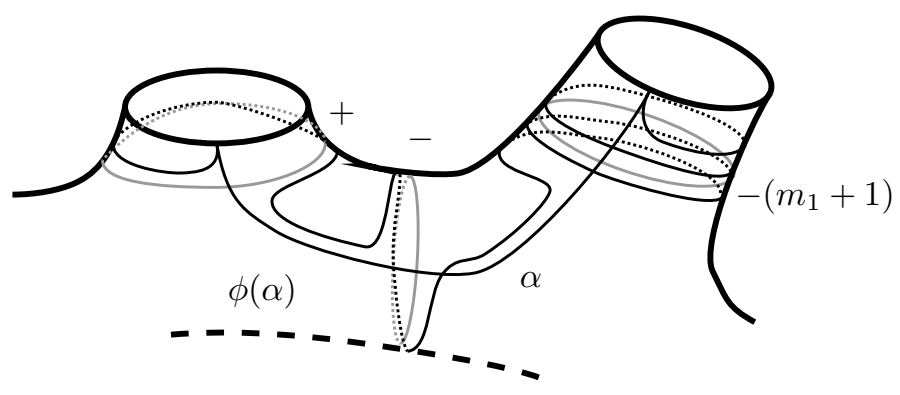

Figure 12: Schematic of the sobering curve

We have shown that $\alpha$ will be sobering in the allowed cases. By theorem 3.4, the presence of a sobering arc implies that the open book is overtwisted. When stabilizing, to get back the open book $(\Sigma(G), \phi(G))$, the compatible contact structure remains overtwisted (in fact, the same up to isotopy), so $(\Sigma(G), \phi(G))$ is overtwisted.

Remark Since they are overtwisted these contact manifolds can't be the convex boundary of a symplectic four-manifold. Corollary 2.1 of [9] also shows 
this for some (but not all) of these graphs. The techniques used there are four-dimensional - a clever application of the adjunction inequality.

\section{References}

[1] V Colin, Chirurgies d'indice un et isotopies de sphères dans les variétés de contact tendues, C. R. Acad. Sci. Paris Sér. I Math. 324 (1997) 659-663 MathReview

[2] R J Daverman, R B Sher (editors), Handbook of geometric topology, NorthHolland, Amsterdam (2002) MathReview

[3] Y Eliashberg, Contact 3-manifolds twenty years since J. Martinet's work, Ann. Inst. Fourier (Grenoble) 42 (1992) 165-192 MathReview

[4] Y M Eliashberg, W P Thurston, Confoliations, University Lecture Series 13, American Mathematical Society, Providence, RI (1998) MathReview

[5] J B Etnyre, Introductory lectures on contact geometry, from: "Topology and geometry of manifolds (Athens, GA, 2001)", Proc. Sympos. Pure Math. 71, Amer. Math. Soc., Providence, RI (2003) 81-107 MathReview

[6] J B Etnyre, K Honda, On the nonexistence of tight contact structures, Ann. of Math. (2) 153 (2001) 749-766 MathReview

[7] J B Etnyre, K Honda, On symplectic cobordisms, Math. Ann. 323 (2002) 31-39 MathReview

[8] D Gabai, Detecting fibred links in $S^{3}$, Comment. Math. Helv. 61 (1986) 519555 MathReview

[9] D T Gay, Open books and configurations of symplectic surfaces, Algebr. Geom. Topol. 3 (2003) 569-586 MathReview

[10] E Giroux, Convexité en topologie de contact, Comment. Math. Helv. 66 (1991) 637-677 MathReview

[11] E Giroux, Géométrie de contact: de la dimension trois vers les dimensions supérieures, from: "Proceedings of the International Congress of Mathematicians, Vol. II (Beijing, 2002)", Higher Ed. Press, Beijing (2002) 405-414 MathReview

[12] E Giroux, N Goodman, On the Stable Equivalence of Open Books in ThreeManifolds, in preparation

[13] N Goodman, Contact Structures and Open Books, PhD thesis, University of Texas at Austin (2003)

[14] K Honda, On the classification of tight contact structures. I, Geom. Topol. 4 (2000) 309-368 MathReview

[15] A Loi, R Piergallini, Compact Stein surfaces with boundary as branched covers of $B^{4}$, Invent. Math. 143 (2001) 325-348 MathReview 
[16] W P Thurston, H E Winkelnkemper, On the existence of contact forms, Proc. Amer. Math. Soc. 52 (1975) 345-347 MathReview

[17] I Torisu, Convex contact structures and fibered links in 3-manifolds, Internat. Math. Res. Notices (2000) 441-454 MathReview

Massachusetts Institute of Technology

Cambridge, MA 02139, USA

Email: ngoodman@math.utexas.edu

Received: 24 July 2004 Revised: 15 June 2005 